\title{
A polymer coating which is sticky yet repulsive to water and slippery yet attractive for oils
}

Received 00th January 20xx, Accepted 00th January 20xx

\author{
Eleanor P. Milnes-Smith, ${ }^{a}$ Corinne A. Stone, ${ }^{b}$ and Susan Perkin *a
}

DOI: $10.1039 / \times 0 \times x 00000 x$

A one-step process has been developed to produce a polymer coating which is hydrophobic and oleophilic, but which oil slides off and water adheres to at all tilt angles - including when vertically inclined or inverted. The polymer is transparent, and the plasma coating process is independent of substrate composition and geometry.

There are many circumstances in which it is desirable to produce a coating which has orthogonal wettabilities for oil and water. Such a surface might be used for oil/water separation, ${ }^{1-4}$ self-cleaning, ${ }^{4}$ anti-fouling,,$^{5-7}$ and anti-fogging purposes $4,6,7$ and can be manufactured using a wide range of techniques. Generally speaking, it is expected that the wetting behaviour of a droplet in motion (dynamic wetting) will be similar to that of a static droplet such that a drop with low adhesion and a high static contact angle (CA) will roll or slide off a surface more easily than a liquid with a low CA. Furthermore, the lower surface energies of hydrocarbons in comparison to water mean that, generally speaking, if water pins to a surface, oil will too. Conversely, if oil slides off, it is likely that water will too. ${ }^{8}$ Dynamic oleo- and hydrophobicity can be a very useful combination, particularly when designing self-cleaning and/or non-fouling surfaces. ${ }^{9-11}$ However, some applications might require orthogonal oil/water sliding behaviour. For example, liquid transport (for lab-on-chip, dying etc.) is most efficient when a larger volume of liquid can be stored on a small area of solid surface without slippage (high static contact angle and strong pinning/dynamic wetting). ${ }^{12,13}$ At the same time, it would desirable to minimize fouling of the surface by other liquids (dynamically de-wetting). This communication provides a description of a one-step procedure for synthesis of a transparent smooth surface coating which is simultaneously hydrophobic yet adhesive for water, and oleophilic yet slippery for oils. . A brief characterization of the

a. Physical \& Theoretical Chemistry Laboratory, Oxford, UK, OX1 3QZ

b. Dstl, Porton Down, Salisbury, Wiltshire UK. SP4 JQ.

+ Footnotes relating to the title and/or authors should appear here.

Electronic Supplementary Information (ESI) available: [details of any supplementary information available should be included here]. See DOI: $10.1039 / x 0 \times x 00000 x$ topography, chemical composition, and wetting properties of the coating is also provided.

Many methods for synthesize surfaces with orthogonal oil/water or static/dynamic behaviour rely on the topography of the surface to physically pin the triple phase line in place. ${ }^{14}$ Here, we present a surface where the special wettability properties are a direct result of the chemical composition although roughness can be useful to enhance the intrinsic properties of a surface. ${ }^{15}$

The surface was synthesized using a low-pressure pulsed plasma polymerization method analogous to a method developed by Coulson et al. for producing fluorinated homopolymers. ${ }^{16}$ There are a number of advantages associated with the use of plasma for surface modification. The lack of solvent means it allows monomers with orthogonal solubilities to be used, such as hydrocarbons and fluorocarbons, and also means that it is a more environmentally friendly method than wet processing. ${ }^{17-19}$ The small amounts of precursor required also adds to its credentials as a green method. The use of vapour also ensures that coverage is uniform and independent of substrate geometry. ${ }^{20}$

The monomers used were 2-hydroxyethyl methacrylate (HEMA) and $1 \mathrm{H}, 1 \mathrm{H}, 2 \mathrm{H}, 2 \mathrm{H}$-perfluorooctyl acrylate (PFAC-6), the structures of which are shown in Figure 1. Previous work has shown that the HEMA and PFAC- 6 homopolymers produced via pulsed plasma polymerization have high monomer conversion and low levels of cross-linking. ${ }^{16,21}$ Monomers were de-gassed by multiple freeze-thaw cycles before use. Substrates to be coated were placed in a glass inductively coupled reactor (a schematic is shown in the supplementary information) and the system was pumped down to base pressure $\left(5 \times 10^{-3} \mathrm{mbar}\right)$. HEMA vapour was introduced at a<smiles>C=CC(=O)OCCC(F)(F)C(F)(F)F</smiles>

Figure 1 (Left) Structure of $1 \mathrm{H}, 1 \mathrm{H}, 2 \mathrm{H}, 2 \mathrm{H}$-perfluorooctyl acrylate "PFAC-6" (Right) Structure of 2-hydroxymethyl methacrylate "HEMA" 


\section{COMMUNICATION}
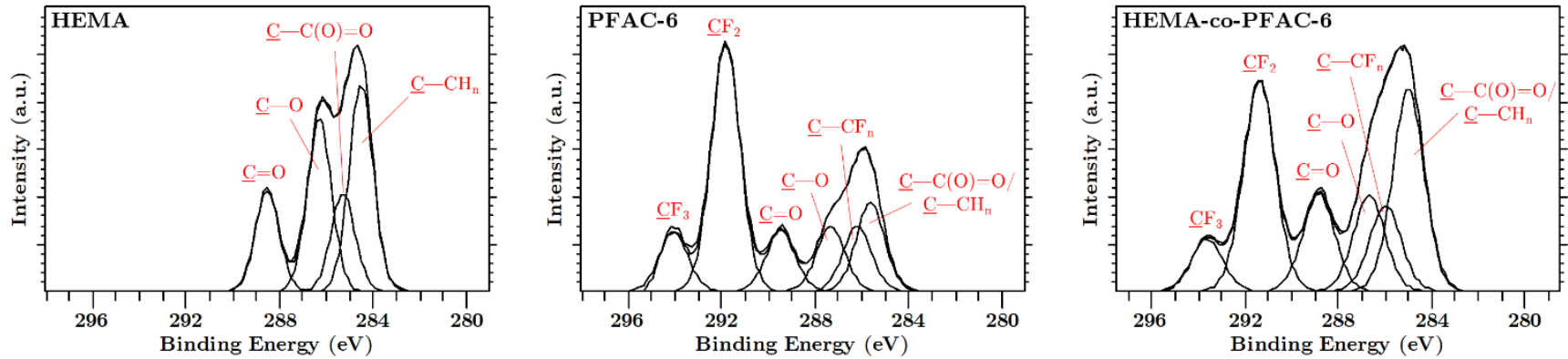

Figure 22 Carbon 1s XPS spectra of (L-R) poly(HEMA), poly(PFAC-6), and poly(HEMA-co-PFAC-6)

pressure of $1 \times 10^{-1}$ mbar and allowed to flow for 5 mins, after which PFAC- 6 vapor was introduced to give a pressure of $2 \mathrm{x}$ $10^{-1}$ mbar. After a further five minutes, the plasma was ignited in pulsed mode at a power of $40 \mathrm{~W}$, and with an 'on' time, $t_{o n}=$ $40 \mu \mathrm{s}$, and 'off'

time, $t_{\text {off }}=20 \mathrm{~ms}$. The total deposition time was $10 \mathrm{~min}$, after which the power was switched off and the vapor allowed to flow for a further 5 min before the system was evacuated to base pressure. For comparison purposes, HEMA and PFAC- 6 homopolymers were also synthesized using the same plasma and pressure parameters as the copolymer. The composition of the surfaces may be altered by adjusting the monomer flow, and the coating thickness may be adjusted by adjusting the deposition time.

The surfaces were characterized using a variety of methods; the chemical composition of the surface was determined using X-ray photoelectron spectroscopy (XPS), the topography of the surface was examined using atomic force microscopy (AFM). The wetting behaviour of the surface was examined using contact angle (CA) goniometry with both static CA's and slide-off angles being measured, the latter with a tilting stage. The probe liquids were $n$-hexadecane $(\mathrm{H})$ and water (W). Further details on characterization methods can be found in the supplementary information.

\begin{tabular}{|l|l|l|l|}
\hline Polymer & \%C & \%O & \%F \\
\hline HEMA & $69 \pm 1(67)$ & $31 \pm 1(33)$ & $1 \pm 1(0)$ \\
\hline PFAC-6 & $43 \pm 1(42)$ & $7 \pm 1(8)$ & $51 \pm 1(50)$ \\
\hline $\begin{array}{l}\text { HEMA-co- } \\
\text { PFAC-6 }\end{array}$ & $47 \pm 1$ & $12 \pm 1$ & $40 \pm 1$ \\
\hline
\end{tabular}

Table 1 Elemental composition (excluding hydrogen) of the polymer surfaces as determined from the XPS survey spectra. The numbers in brackets are the predicted values for the intact polymer with no crosslinking/fragmentation, and randomly oriented monomer units. The co-monomer ration was calculated using these values
The compositions of the coating and of the HEMA and PFAC- 6 homopolymers were determined by examining the relative areas under the $C(1 s), F(1 s)$, and $O(1 s)$ peaks in the XPS survey spectra and are shown in Table 1 . This shows that the surface is composed of 17-22 mol\% HEMA, depending on which element is used for benchmarking. The lack of peaks associated with silicon indicates that the glass substrate is completely covered. Deconvoluted $\mathrm{C}(1 \mathrm{~s})$ spectra are shown in Figure 2 and a table of peak positions is given in the supplementary information. Although cross-linking and fragmentation processes cannot be entirely eliminated, the extremely short duty cycle of the pulsed process means that the amounts of cross-linked and/or fragmented polymer are very small, and the polymers product has a high level of structural retention. This conclusion is supported by the fact the HEMA and PFAC-6 plasma polymers produced via this method have an identical chemical composition to the pure homopolymers, and by the apparent lack of peaks associated with cross-linked carbon in the $\mathrm{C}(1 \mathrm{~s})$ spectra in Figure 2.

XPS does not distinguish between a composite surface and a true copolymer. However, recent work by Fouquet et al. has shown that fluorocarbon and hydrocarbon acrylates copolymerize in the plasma phase ${ }^{18}$ and the acrylate and methacrylate moieties are sufficiently similar to assume that a HEMA-co-PFAC- 6 copolymer has been formed. In the context of a copolymer, there also arises the question as to whether a statistical copolymer is formed or whether nanodomains of homopolymer are formed. Amadei et al. investigated a copolymer of HEMA and $1 \mathrm{H}, 1 \mathrm{H}, 2 \mathrm{H}, 2 \mathrm{H}$-perfluorodecyl acrylate (PFAC-8) synthesized via iCVD and found nanodomains of homopolymer between 1-2 $\mathrm{nm}^{2}$ in size. ${ }^{22}$ However, the difference in phase behaviour between eight and six membered perfluoroalkyl chains (solid and liquid respectively at room temperature) means that it is uncertain as to whether HEMA-Co-PFAC- 6 would behave in the same way. ${ }^{23}$ 

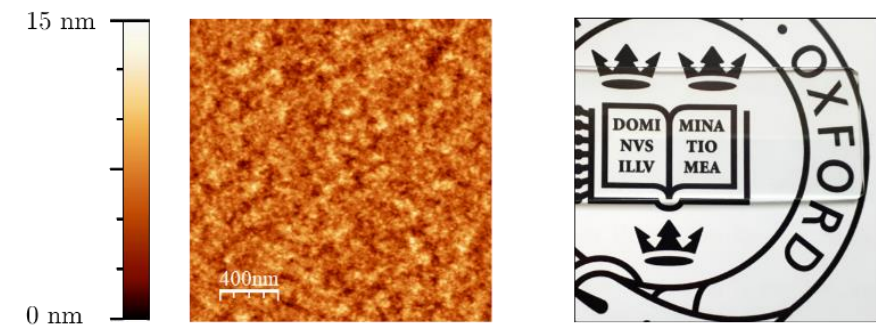

Figure 3 Left) $2 \times 2 \mu \mathrm{m} 2$ AFM micrograph of poly(HEMA-co-PFAC-6) deposited onto glass (Right) Photograph of a glass slide coated with poly(HEMA-coPFAC-6) coating

AFM measurements showed that the copolymer coating is uniform across the substrate and relatively smooth, with an RMS roughness of $1.7 \mathrm{~nm}$. An example micrograph is shown in Figure 3. In comparison, the HEMA homopolymer has an RMS roughness of $0.8 \mathrm{~nm}$, which is close to the noise level of the instrument. The PFAC- 6 homopolymer is a viscous liquid at room temperature and was therefore too soft to acquire meaningful topographical measurements. It is interesting to note that the incorporation of HEMA into the PFAC- 6 polymer results in a solid surface.

On a macroscopic scale, the copolymer coating is optically transparent and performs well in the scotch tape test although it may be scratched with a scalpel. Figure 3 demonstrates the transparency of the coating on glass.

In terms of static CA's, poly(HEMA) is both hydrophilic and oleophilic with complete spreading of both liquids whilst poly(PFAC-6) is both hydrophobic (WCA $=121 \pm 9^{\circ}$ ) and moderately oleophobic $\left(\mathrm{HCA}=98 \pm 3^{\circ}\right)$. The copolymer is hydrophobic (WCA $=110 \pm 1^{\circ}$ ) and moderately oleophilic (HCA $=75 \pm 2^{\circ}$ ). Errors are standard deviations arising from measurements on three surfaces prepared independently (separate plasma cycles) and five measurements performed on each surface. Experiments using a tilting stage showed that oil and water was pinned to poly(PFAC-6) at all angles, whilst the spreading of both liquids on poly(HEMA) precluded any measurements on this surface. In contrast, oil drops slid off the copolymer surface at a tilt angle of $20^{\circ}$ leaving no residue, whilst water drops pinned to the surface at all tilt angles - as shown in Figure 4.

The low roughness of all of the surfaces would indicate that the wetting behaviour of the copolymer is an intrinsic, chemical property of the surface, rather than a consequence of pinning on topographical features. The unusual wetting behaviour of the poly(PFAC-6) (high contact angles and strong pinning) is a result of surface reorganization; in air, the fluorinated side chains are at the surface whilst contact with liquid leads to migration of the acrylate groups to the surface. ${ }^{23}$ We propose that the incorporation of HEMA into poly(PFAC-6) leads to hydrogen bonding between the hydroxyl group in HEMA and the carbonyl groups in the acrylate or methacrylate groups, inhibiting surface reorganization and triggering the transition from liquid to solid. Depositing water on the surface would disrupt the hydrogen bonding interaction and allow surface reorganization to take place (pinning the water drop in place) whilst oil would have no such effect and can move freely across the surface. It should be noted that this
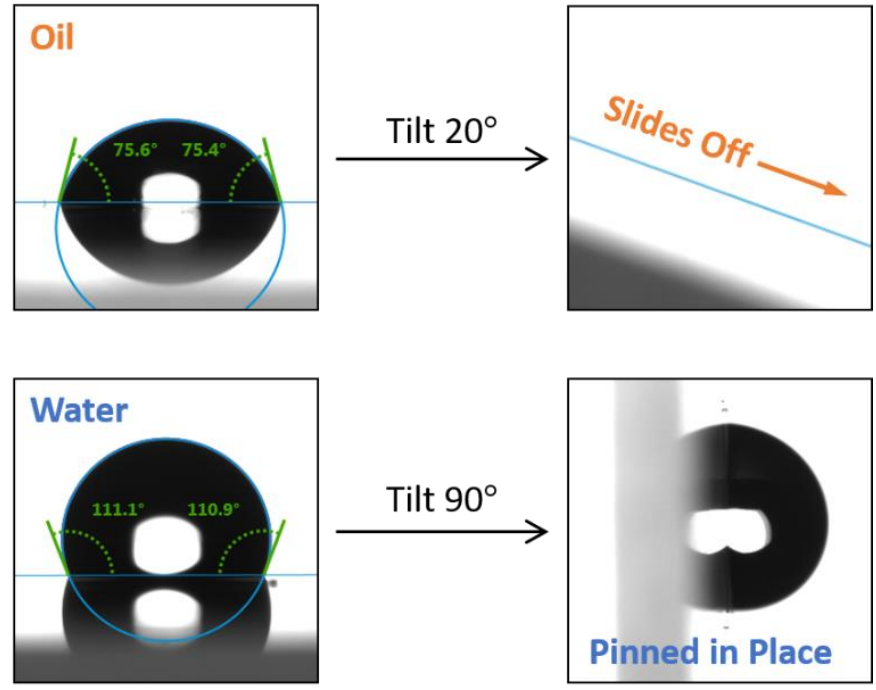

Figure 4 Static (left) and dynamic (right) behaviour of $n$-hexadecane (top) and water (bottom) on poly(HEMA-co-PFAC-6) surfaces

hydrogen bonding mechanism is only possible in the context of a true copolymer, rather than a mix of homopolymers.

In conclusion, a HEMA-Co-PFAC-6 polymer coating has been synthesized which is hydrophobic and moderately oleophilic but which water sticks to and oil slides off. The synthesis was via pulsed plasma polymerization, a method already used in industry to produce a polymer and graft it to a substrate of choice in a single step. The method has the additional advantage in that no regulation of monomer flow rate is needed. The results shown here are preliminary measurements and further work is needed to examine the wetting behaviour of a wider range of liquids and the response to stimuli such as changes in temperature, $\mathrm{pH}$, and immersion in oil or water. ${ }^{24,} 25$ The properties of the HEMA and PFAC- 6 homopolymers provide a useful benchmark of the two extremes of wetting behaviour and it is likely that the properties of the copolymer could be tailored extensively by adjusting the monomer ratios and substrate topography.

\section{Conflicts of interest}

There are no conflicts to declare.

\section{Acknowledgements}

The research was supported by the European Research Council (under Starting Grant No. 676861, LIQUISWITCH).

\section{Notes and references}

1. Z. Chu, Y. Feng and S. Seeger, Angewandte Chemie International Edition, 2015, 54, 2328-2338.

2. Q. Ma, H. Cheng, A. G. Fane, R. Wang and H. Zhang, Small, 2016, 12, 2186-2202.

3. Z. Xue, Y. Cao, N. Liu, L. Feng and L. Jiang, Journal of Materials Chemistry A, 2014, 2, 2445-2460. 
4. P. S. Brown, O. D. L. A. Atkinson and J. P. S. Badyal, ACS Applied Materials \& Interfaces, 2014, 6, 7504-7511.

5. S. Hutton, J. Crowther and J. Badyal, Chemistry of Materials, 2000, 12, 2282-2286.

6. J. A. Howarter and J. P. Youngblood, Advanced Materials, 2007, 19, 3838-3843.

7. J. A. Howarter and J. P. Youngblood, Macromolecular Rapid Communications, 2008, 29, 455-466.

8. A. Tuteja, W. Choi, M. Ma, J. M. Mabry, S. A. Mazzella, G. C. Rutledge, G. H. McKinley and R. E. Cohen, Science, 2007, 318, 1618-1622.

9. D. F. Cheng, C. Urata, M. Yagihashi and A. Hozumi, Angewandte Chemie International Edition, 2012, 51, 2956-2959.

10. L. Zhang, Y. Zhong, D. Cha and P. Wang, Scientific Reports, 2013, 3, 2326 .

11. M. Nosonovsky and B. Bhushan, Current Opinion in Colloid \& Interface Science, 2009, 14, 270-280.

12. N. Zhao, Q. Xie, X. Kuang, S. Wang, Y. Li, X. Lu, S. Tan, J. Shen, X. Zhang, Y. Zhang, J. Xu and C. C. Han, Advanced Functional Materials, 2007, 17, 2739-2745.

13. X. Song, J. Zhai, Y. Wang and L. Jiang, The Journal of Physical Chemistry B, 2005, 109, 4048-4052.

14. D. Quere, Annual Review of Materials Research, 2008, 38, 71-99.

15. R. N. Wenzel, Industrial \& Engineering Chemistry, 1936, 28, 988-994.

16. S. R. Coulson, I. S. Woodward, J. P. S. Badyal, S. A. Brewer and C. Willis, Chemistry of Materials, 2000, 12, 20312038.

17. H. Yasuda and Y. Matsuzawa, Plasma Processes and Polymers, 2005, 2, 507-512.

18. T. Fouquet, G. Mertz, M. Delmée, C. Becker, J. Bardon and H. Sato, Plasma Processes and Polymers, 2016, 13, 862868.

19. C. S. Gudipati, J. A. Finlay, J. A. Callow, M. E. Callow and K. L. Wooley, Langmuir, 2005, 21, 3044-3053.

20. C. M. Chan, T. M. Ko and H. Hiraoka, Surface Science Reports, 1996, 24, 1-54.

21. C. Tarducci, W. C. E. Schofield, J. P. S. Badyal, S. A. Brewer and C. Willis, Chemistry of Materials, 2002, 14, 2541 2545.

22. C. A. Amadei, R. Yang, M. Chiesa, K. K. Gleason and S. Santos, ACS Applied Materials \& Interfaces, 2014, 6, 47054712.

23. K. Honda, M. Morita, H. Otsuka and A. Takahara, Macromolecules, 2005, 38, 5699-5705.

$24 . \quad$ B. Wang, W. Liang, Z. Guo and W. Liu, Chemical Society Reviews, 2015, 44, 336-361.

25. D. Parbat, S. Gaffar, A. M. Rather, A. Gupta and U. Manna, Chemical science, 2017, 8, 6542-6554. 